\title{
The Almost Sure Asymptotic Stability and Boundedness of Stochastic Functional Differential Equations with Polynomial Growth Condition
}

\author{
Lichao Feng ${ }^{1,2}$ and Shoumei $\mathrm{Li}^{1}$ \\ ${ }^{1}$ College of Applied Science, Beijing University of Technology, Beijing 100124, China \\ ${ }^{2}$ College of Science, Hebei United University, Tangshan, Hebei 063009, China
}

Correspondence should be addressed to Shoumei Li; lishoumei2011@gmail.com

Received 9 May 2014; Revised 2 August 2014; Accepted 4 August 2014; Published 18 August 2014

Academic Editor: Daoyi Xu

Copyright (C) 2014 L. Feng and S. Li. This is an open access article distributed under the Creative Commons Attribution License, which permits unrestricted use, distribution, and reproduction in any medium, provided the original work is properly cited.

\begin{abstract}
Stability and boundedness are two of the most important topics in the study of stochastic functional differential equations (SFDEs). This paper mainly discusses the almost sure asymptotic stability and the boundedness of nonlinear SFDEs satisfying the local Lipschitz condition but not the linear growth condition. Here we assume that the coefficients of SFDEs are polynomial or dominated by polynomial functions. We give sufficient criteria on the almost sure asymptotic stability and the boundedness for this kind of nonlinear SFDEs. Some nontrivial examples are provided to illustrate our results.
\end{abstract}

\section{Introduction}

Stochastic modeling plays an important role in many branches of sciences and industries. Since Itô introduced his stochastic calculus, stochastic delay or functional differential equations (SDDEs or SFDEs) have been used successfully to model those systems which depend not only on the present history of the state but also on the past ones (see, e.g., [1-5]). Stability and boundedness are two of the most important topics in the study of SDDEs or SFDEs in modern control theory. Many researchers have done a lot of works for these two topics (see, e.g., [6-18]).

In general, a SFDE has the form

$$
\mathrm{d} \mathbf{x}(t)=\mathbf{f}\left(\mathbf{x}_{t}, t\right) \mathrm{d} t+\mathbf{g}\left(\mathbf{x}_{t}, t\right) \mathrm{d} B(t)
$$

on $t \geq 0$ with initial data $\zeta \in C_{\mathscr{F}_{0}}^{b}\left([-\tau, 0] ; R^{n}\right)$, where f : $C\left([-\tau, 0] ; R^{n}\right) \times R_{+} \rightarrow R^{n}$ and $\mathbf{g}: C\left([-\tau, 0] ; R^{n}\right) \times$ $R_{+} \rightarrow R^{n \times m}$ (the notations used here will be illustrated in Section 2). Most of the existing stability criteria of SFDEs require the coefficients of corresponding systems to satisfy the local Lipschitz condition and the linear growth condition or the one-side linear growth condition (see, e.g., $[2,4,5]$ ). However, many SDDEs or SFDEs can not be dominated by the linear growth condition, such as stochastic population system, Lotka-Volterra systems, and system (2) as follows:

$$
\begin{aligned}
\mathrm{d} \mathbf{x}(t)= & \left(-4 \mathbf{x}^{3}(t)-3 \mathbf{x}(t)+2 D_{1}^{2}\left(\mathbf{x}_{t}\right)\right) \mathrm{d} t \\
& +\left(\mathbf{x}^{5 / 3}(t)+D_{2}\left(\mathbf{x}_{t}\right)\right) \mathrm{d} B(t)
\end{aligned}
$$

with initial data $\zeta \in C_{\mathscr{F}_{0}}^{b}\left([-\tau, 0] ; R^{n}\right)$, where $B(t)$ is a scalar Brownian motion, $D_{i}$ are bounded linear operators from $C([-\tau, 0] ; R)$ to $R$ satisfying $\left|D_{i}\left(\mathbf{x}_{t}\right)\right| \leq \int_{-\tau}^{0}|\mathbf{x}(t+\theta)| \mathrm{d} \eta_{i}(\theta)$, and $\eta_{i}(\cdot)$ are probability measures on $[-\tau, 0], i=1,2$.

So it is necessary to consider the cases of the nonlinear growth condition. Recently, Liu et al. [10] study the asymptotic stability of nonlinear stochastic differential equations (SDEs) with polynomial growth condition, and they also develop their results to the case of SDDEs [11]. In this paper, we mainly establish some new results on the almost sure asymptotic stability and the boundedness in the sense of the pth moment and the trajectory with large probability of SFDEs with polynomial growth condition, which imply the results in $[10,11]$.

Here we would like to mention the work of Luo et al. [12]. It proposes a generalized theory for the asymptotic stability 
and the boundedness for SFDEs based on a Lyapunovtype condition without the linear growth condition; more precisely, the diffusion operator of a Lyapunov function $V$ is required to satisfy the following condition:

$$
\begin{aligned}
\mathscr{L} V(\varphi, t) \leq & a_{1}-a_{2} V(\varphi(0), t) \\
& +a_{3} \int_{-\tau}^{0} V(\varphi(\theta), t+\theta) \mathrm{d} \widetilde{\mu}(\theta)-U(\varphi(0), t) \\
& +a \int_{-\tau}^{0} U(\varphi(\theta), t+\theta) \mathrm{d} \mu(\theta)
\end{aligned}
$$

where $\tilde{\mu}$ and $\mu$ are probability measures on $[-\tau, 0], a_{1} \geq$ $0, a_{2}>a_{3} \geq 0, a \in(0,1)$. However as to system (2), setting $V(\mathbf{x}, t)=|\mathbf{x}|^{2}$, we can get

$$
\begin{aligned}
\mathscr{L} V(\boldsymbol{\varphi}, t)= & -8|\boldsymbol{\varphi}(0)|^{4}-4|\boldsymbol{\varphi}(0)|^{2}+2 \int_{-\tau}^{0}|\boldsymbol{\varphi}(\theta)|^{4} \mathrm{~d} \eta_{1}(\theta) \\
& +\left(|\boldsymbol{\varphi}(0)|^{5 / 3}+\int_{-\tau}^{0}|\varphi(\theta)| \mathrm{d} \eta_{2}(\theta)\right)^{2} \\
\leq & -8|\varphi(0)|^{4}-4|\varphi(0)|^{2}+2 \int_{-\tau}^{0}|\varphi(\theta)|^{4} \mathrm{~d} \eta_{1}(\theta) \\
& +2|\varphi(0)|^{10 / 3}+2 \int_{-\tau}^{0}|\varphi(\theta)|^{2} \mathrm{~d} \eta_{2}(\theta)
\end{aligned}
$$

Since the above $\mathscr{L} V(\varphi, t)$ includes the positive term $|\varphi(0)|^{10 / 3}$, it does not satisfy (3). So their work does not imply ours. Also Shen et al. [13] use the LaSall technique to study the almost sure asymptotical stability of SFDEs under different settings.

The organization of this paper is as follows: Section 2 describes some necessary notations and lemmas; the existence of the global solution and the bounedness of SFDEs are stated in Section 3; sufficient conditions are proposed for the almost sure asymptotic stability in Section 4; to show the applications of our results, some illustrative examples are given in the final section.

\section{Preliminaries}

Through this paper, let $\left(\Omega, \mathscr{F},\left\{\mathscr{F}_{t}\right\}_{t \geq 0}, \mathbb{P}\right)$ be a complete probability space with a filtration $\left\{\mathscr{F}_{t}\right\}_{t \geq 0}$ satisfying the usual conditions and $B(t)$ an $m$-dimensional Brownian motion defined on the probability space. Let $\tau>0$ and let $C\left([-\tau, 0] ; R^{n}\right)$ denote the family of all continuous $R^{n}$-valued functions $\varphi$ on $[-\tau, 0]$ with the norm $\|\varphi\|=\sup _{-\tau \leq \theta \leq 0}|\varphi(\theta)|$. Let $C=C_{\mathscr{F}_{0}}^{b}\left([-\tau, 0] ; R^{n}\right)$ be the family of all bounded, $\mathscr{F}_{0}$-measurable, $C\left([-\tau, 0] ; R^{n}\right)$-valued, $\mathscr{F}_{t}$-adapted stochastic processes. Let $\eta_{i}$ be probability measures on $[-\tau, 0]$, which satisfy $\int_{-\tau}^{0} d \eta_{i}(\theta)=1(i=1,2,3,4)$. Let $L^{1}\left(R_{+} ; R_{+}\right)$be the family of all functions $\xi: R_{+} \rightarrow R_{+}$such that $\int_{0}^{+\infty} \xi(t) \mathrm{d} t<$ $\infty$. $\mathbf{x}(t)$ is a continuous $R$-valued stochastic process on $t \in$ $[-\tau, \infty)$. We assume $\mathbf{x}_{t}=\{\mathbf{x}(t+\theta):-\tau \leqslant \theta \leqslant 0\}$ for all $t \geq 0$, which is regarded as a $C\left([-\tau, 0] ; R^{n}\right)$-valued stochastic process.

Consider an $n$-dimensional SFDE

$$
\mathrm{d} \mathbf{x}(t)=\mathbf{f}\left(\mathbf{x}_{t}, t\right) \mathrm{d} t+\mathbf{g}\left(\mathbf{x}_{t}, t\right) \mathrm{d} B(t),
$$

on $t \geq 0$ with initial data $\{\mathbf{x}(\theta):-\tau \leq \theta \leq 0\}=\zeta \epsilon$ $C_{\mathscr{F}_{0}}^{b}\left([-\tau, 0] ; R^{n}\right)$, where

$$
\begin{gathered}
\mathbf{f}: C\left([-\tau, 0] ; R^{n}\right) \times R_{+} \longrightarrow R^{n} \\
\mathbf{g}: C\left([-\tau, 0] ; R^{n}\right) \times R_{+} \longrightarrow R^{n \times m}
\end{gathered}
$$

Assume furthermore that $\mathbf{f}(0, t)=0$ and $\mathbf{g}(0, t)=0$, so system (5) has the solution $\mathbf{x}(t)=0$. The solution is called the trial solution or equilibrium solution.

To get our main results, we firstly put forward the following hypothesis.

Assumption 1 (the local Lipschitz condition). For each integer $k=1,2, \ldots$, there exists a positive constant $\mathrm{d}_{k}>0$ such that

$$
|\mathbf{f}(\boldsymbol{\varphi}, t)-\mathbf{f}(\psi, t)| \vee|\mathbf{g}(\boldsymbol{\varphi}, t)-\mathbf{g}(\psi, t)| \leq \mathrm{d}_{k}\|\boldsymbol{\varphi}-\psi\|,
$$

for all $\varphi, \psi \in C\left([-\tau, 0] ; R^{n}\right), t \in R_{+}$with $\|\varphi\| \vee\|\psi\| \leq k$.

Remark 2. By Theorem 3.1 in [15] or Lemma 2.3 in [16], this assumption with conditions $\mathbf{f}(0, t)=0$ and $\mathbf{g}(0, t)=0$ can guarantee a unique maximal local solution to system (5) for any initial data.

However, to ensure the unique maximal local solution is in fact the global solution, we need to impose the following additional polynomial growth condition.

Assumption 3 (the polynomial growth condition). There exist constants $\kappa, \bar{\kappa}, \underline{\kappa}, \gamma \geq 0$, probability measures $\eta_{i}$ on $[-\tau, 0], i=$ $1,2,3,4$, and positive numbers $n_{1}>1, n_{2}>1$ satisfying $n_{1}+$ $1>2 n_{2}$, and bounded functions $\xi_{1}(t), \xi_{2}(t) \in L^{1}\left(R_{+} ; R_{+}\right)$such that

$$
\begin{array}{r}
\boldsymbol{\varphi}(0)^{T} \mathbf{f}(\boldsymbol{\varphi}, t) \leq-\kappa|\boldsymbol{\varphi}(0)|^{n_{1}+1}+\bar{\kappa} \int_{-\tau}^{0}|\boldsymbol{\varphi}(\theta)|^{n_{1}+1} d \eta_{1}(\theta) \\
-|\boldsymbol{\varphi}(0)|^{2}+\underline{\kappa} \int_{-\tau}^{0}|\boldsymbol{\varphi}(\theta)|^{2} d \eta_{2}(\theta)+\xi_{1}(t), \\
|\mathbf{g}(\boldsymbol{\varphi}, t)| \leq \gamma\left(|\boldsymbol{\varphi}(0)|^{n_{2}}+\int_{-\tau}^{0}|\boldsymbol{\varphi}(\theta)|^{n_{2}} d \eta_{3}(\theta)+|\boldsymbol{\varphi}(0)|\right. \\
\left.+\int_{-\tau}^{0}|\boldsymbol{\varphi}(\theta)| d \eta_{4}(\theta)\right)+\xi_{2}(t),
\end{array}
$$

for all $\varphi \in C\left([-\tau, 0] ; R^{n}\right), t \in R_{+}$.

Remark 4. The probability measures $\eta_{i}, i=1,2,3,4$, can be weakened to any right continuous nondecreasing functions (see [19]). Compared with [10, 11], Assumption 3 in this paper is a generalization of Assumption 3 of [10] and Assumption 2 of [11]. 
Let $C^{2,1}\left(R^{n} \times[-\tau,+\infty) ; R_{+}\right)$denote the family of all continuous nonnegative functions $V(x, t)$ on $R^{n} \times[-\tau,+\infty)$, which are continuously twice differentiable in $x$ and once differentiable in $t$. For each $V \in C^{2,1}\left(R^{n} \times[-\tau,+\infty) ; R_{+}\right)$, denote an operator $\mathscr{L} V$ from $C\left([-\tau, 0] ; R^{n}\right) \times R_{+}$to $R$ by

$$
\begin{aligned}
\mathscr{L} V(\boldsymbol{\varphi}, t)= & V_{t}(\boldsymbol{\varphi}(0), t)+V_{x}(\boldsymbol{\varphi}(0), t) \mathbf{f}(\boldsymbol{\varphi}, t) \\
& +\frac{1}{2} \operatorname{trace}\left[\mathbf{g}^{T}(\boldsymbol{\varphi}, t) V_{x x}(\boldsymbol{\varphi}(0), t) \mathbf{g}(\boldsymbol{\varphi}, t)\right],
\end{aligned}
$$

where $V_{t}(\mathbf{x}, t)=\partial V(\mathbf{x}, t) / \partial t, V_{x x}(\mathbf{x}, t)=\left(\partial^{2} V(\mathbf{x}, t) /\right.$ $\left.\partial x_{i} x_{j}\right)_{n \times n}$, and $V_{x}(\mathbf{x}, t)=\left(\partial V(\mathbf{x}, t) / \partial x_{1}, \ldots, \partial V(\mathbf{x}, t) / \partial x_{n}\right)$.

Then let us recall a number of lemmas.

Lemma 5 (cf. [20]). If $h(t)$ is a bounded function on $[0, \infty)$ and $h(t) \in L^{1}\left(R_{+} ; R_{+}\right)$, then for any $\beta \geq 1, \int_{0}^{+\infty} h^{\beta}(t) d t<\infty$.

Lemma 6 (cf. [11]). Assume $a, b, q>0, b \geq q, \alpha>\beta>0$. If the following condition holds,

$$
\frac{a}{b}>(\alpha-\beta)^{\beta /(\alpha-\beta)} \beta \alpha^{-\alpha /(\alpha-\beta),}
$$

then there exists $\bar{a} \in(0, a)$ satisfying

$$
a+b t^{\alpha}-q t^{\beta}>\bar{a},
$$

for all $t \geq 0$.

Lemma 7 (cf. [14]). Assume $\alpha, \beta>0$. For any $h(t) \in C\left(R^{n}\right.$; $R)$, if $\lim \sup _{|t| \rightarrow \infty}\left(h(t) /|t|^{\alpha}\right)=0$, then there exists a constant $H$ satisfying

$$
\sup _{t \in R^{n}}\left\{-\beta|t|^{\alpha}+h(t)\right\}<H .
$$

Lemma 8 (Kolmogorov-Chentsov theorem [21]). Suppose that a stochastic process $X(t)$ on $t \geq 0$ satisfies the condition

$$
E|X(t)-X(s)|^{\alpha} \leq D|t-s|^{1+\beta}, \quad 0 \leq s, t<\infty
$$

for some positive constants $\alpha, \beta$, and $D$. Then there exists a continuous modification $\widetilde{X}(t)$ of $X(t)$, which has the property that, for every $\gamma \in(0, \beta / \alpha)$, there is a positive random variable $\delta(\omega)$ such that

$$
P\left\{\omega: \sup _{0<t-s<\delta(\omega), 0 \leq s, t<\infty} \frac{|\widetilde{X}(t, \omega)-\widetilde{X}(s, \omega)|}{|t-s|^{\gamma}} \leq \frac{2}{1-2^{\gamma}}\right\}=1 .
$$

In other words, almost every sample path of $\widetilde{X}(t)$ is locally but uniformly Hölder-continuous with exponent $\gamma$.

\section{Boundedness of SFDEs}

For a stochastic differential equation to have a unique global solution for any given initial value, the coefficients of this equation are generally required to satisfy the linear growth condition and the local Lipschitz condition (see $[2,4,5]$ ) or a given non-Lipschitz condition and the linear growth condition (see [22]). However, when the coefficients of the system (5) satisfy the local Lipschitz condition and the polynomial growth condition, the solution of the system (5) may explode at a finite time. So it is necessary to examine the existence and uniqueness of the global solution of the system (5). Here we state the following existence-and-uniqueness result.

Lemma 9. If Assumptions 1 and 3 and $\kappa>\bar{\kappa}$ hold, then for any initial data $\zeta \in C_{\mathscr{F}_{0}}^{b}\left([-\tau, 0] ; R^{n}\right)$, there is a unique global solution $\mathbf{x}(t, \zeta)$ of system (5) on $t \geq-\tau$.

Remark 10. This result is the special case of Theorem 3.2 of [15]. Since it is not so easy to see this fact directly, we give the proof in the Appendix. The fact that we write down our Lemma 9 here is to keep our paper completely based on Assumptions 1 and 3.

We now show the following asymptotic boundedness of the global solution in the sense of the pth moment and the trajectory with large probability.

Theorem 11. If Assumptions 1 and 3 and $\kappa>\bar{\kappa}$ hold, then for any initial data $\zeta \in C$ and any $p \geq 0$, the global solution $\mathbf{x}(t, \zeta)$ of system (5) is bounded in the sense of pth moment; that is, there exists a constant $M_{p}>0$ such that

$$
\sup _{-\tau \leq t<+\infty} E|\mathbf{x}(t, \zeta)|^{p} \leq M_{p} .
$$

Proof. Since $\kappa>\bar{\kappa}$, the existence and uniqueness of the solution follow from Lemma 9. And there exists at least a sufficiently small positive constant $\varepsilon$ satisfying $\kappa>\bar{\kappa} e^{\varepsilon \tau}$. So by the continuity, define $\varepsilon^{\prime \prime}=\sup \left\{\varepsilon>0: \kappa>\bar{\kappa} e^{\varepsilon \tau}\right\}$. For the sake of simplicity, write $\mathbf{x}(t)=\mathbf{x}(t, \zeta), \mathbf{x}_{t}=\mathbf{x}_{t}^{\zeta}$. For any $p \geq 2$, applying Itô's formula to $V(\mathbf{x}, t)=e^{\varepsilon t}|\mathbf{x}(t)|^{p}, \varepsilon \in\left(0, \varepsilon^{\prime \prime}\right]$, we yield

$$
\begin{aligned}
& \mathscr{L} V(\mathbf{x}, t) \\
& =e^{\varepsilon t}\left(\mathscr{L}|\mathbf{x}(t)|^{p}+\varepsilon|\mathbf{x}(t)|^{p}\right) \\
& \leq e^{\varepsilon t}\left[\frac{p}{2}|\mathbf{x}(t)|^{p-2}\right. \\
& \quad \times\left(2 \mathbf{x}^{T}(r) \mathbf{f}\left(\mathbf{x}_{r}, r\right)+(p-1)\left|\mathbf{g}\left(\mathbf{x}_{r}, r\right)\right|^{2}\right) \\
& \left.\quad+\varepsilon|\mathbf{x}(t)|^{p}\right] \\
& \leq e^{\varepsilon t} \frac{p}{2}|\mathbf{x}(t)|^{p-2} \\
& \times\left[2 \left(-\kappa|\mathbf{x}(t)|^{n_{1}+1}+\bar{\kappa} \int_{-\tau}^{0}|\mathbf{x}(t+\theta)|^{n_{1}+1} d \eta_{1}(\theta)\right.\right. \\
& \left.\quad-|\mathbf{x}(t)|^{2}+\underline{\kappa} \int_{-\tau}^{0}|\mathbf{x}(t+\theta)|^{2} d \eta_{2}(\theta)+\xi_{1}(t)\right) \\
& \quad+5(p-1)
\end{aligned}
$$




$$
\begin{gathered}
\times\left(\gamma ^ { 2 } \left[|\mathbf{x}(t)|^{2 n_{2}}+\int_{-\tau}^{0}|\mathbf{x}(t+\theta)|^{2 n_{2}} d \eta_{3}(\theta)\right.\right. \\
\left.+|\mathbf{x}(t)|^{2}+\int_{-\tau}^{0}|\mathbf{x}(t+\theta)|^{2} d \eta_{4}(\theta)\right] \\
\left.\left.+\xi_{2}^{2}(t)\right)+\frac{2}{p} \varepsilon|\mathbf{x}(t)|^{2}\right] \\
\leq \frac{p}{2} e^{\varepsilon t}\left[-2\left(\kappa-\bar{\kappa} e^{\varepsilon \tau}\right)|\mathbf{x}(t)|^{p+n_{1}-1}+o\left(|\mathbf{x}(t)|^{p+n_{1}-1}\right)\right] \\
+2 \underline{\kappa} e^{\varepsilon t} J_{2}+5(p-1) \gamma^{2} e^{\varepsilon t} J_{4}+\frac{p}{2} 5(p-1) \gamma^{2} \\
\times \frac{2 n_{2}}{p+2 n_{2}-2} e^{\varepsilon t} J_{3}+p \bar{\kappa} \frac{n_{1}+1}{p+n_{1}-1} e^{\varepsilon t} J_{1}+2 e^{\varepsilon t} \xi_{1}^{p / 2}(t) \\
+5(p-1) e^{\varepsilon t} \xi_{2}^{p}(t),
\end{gathered}
$$

where $J_{1}=\int_{-\tau}^{0}|\mathbf{x}(t+\theta)|^{p+n_{1}-1} \mathrm{~d} \eta_{1}(\theta)-e^{\varepsilon \tau}|\mathbf{x}(t)|^{p+n_{1}-1}, J_{2}=$ $\int_{-\tau}^{0}|\mathbf{x}(t+\theta)|^{p} \mathrm{~d} \eta_{2}(\theta)-e^{\varepsilon \tau}|\mathbf{x}(t)|^{p}, J_{3}=\int_{-\tau}^{0}|\mathbf{x}(t+\theta)|^{p+2 n_{2}-2} \mathrm{~d} \eta_{3}$ $(\theta)-e^{\varepsilon \tau}|\mathbf{x}(t)|^{p+2 n_{2}-2}$, and $J_{4}=\int_{-\tau}^{0}|\mathbf{x}(t+\theta)|^{p} \mathrm{~d} \eta_{4}(\theta)-e^{\varepsilon \tau}|\mathbf{x}(t)|^{p}$.

Noting that $\kappa>\bar{\kappa} e^{\varepsilon \tau}$ and $|\mathbf{x}(t)| \geq 0$ for any $t \geq 0$, by Lemma 7 and the same technique as (A.3), $G(|\mathbf{x}(t)|)=$ $-2\left(\kappa-\bar{\kappa} e^{\varepsilon \tau}\right)|\mathbf{x}(t)|^{p+n_{1}-1}+o\left(|\mathbf{x}(t)|^{p+n_{1}-1}\right)$, as a function of $|\mathbf{x}(t)|$, has a positive upper-boundedness; that is, there is a positive constant $Q$ such that

$G(|\mathbf{x}(t)|)=-2\left(\kappa-\bar{\kappa} e^{\varepsilon \tau}\right)|\mathbf{x}(t)|^{p+n_{1}-1}+o\left(|\mathbf{x}(t)|^{p+n_{1}-1}\right) \leq Q$.

And in view of the fact that

$$
\begin{aligned}
& \int_{0}^{t} e^{\varepsilon s} J_{i} \mathrm{~d} s \\
& \quad=\int_{0}^{t} e^{\varepsilon s}\left(\int_{-\tau}^{0}|\mathbf{x}(s+\theta)|^{w_{i}} \mathrm{~d} \eta_{i}(\theta)-e^{\varepsilon \tau}|\mathbf{x}(s)|^{w_{i}}\right) \mathrm{d} s \\
& \quad \leq e^{\varepsilon \tau} \int_{-\tau}^{0}|\mathbf{x}(s)|^{w_{i}} \mathrm{~d} s,
\end{aligned}
$$

for $w_{1}=p+n_{1}-1, w_{3}=p+2 n_{2}-2$, and $w_{2}=w_{4}=p$, we yield that, for $t \geq 0$,

$$
\begin{aligned}
E e^{\varepsilon t}|\mathbf{x}(t)|^{p} \leq & E|\mathbf{x}(0)|^{p}+\frac{p}{2} Q \int_{0}^{t} e^{\varepsilon s} \mathrm{~d} s \\
& +p \bar{\kappa} \frac{n_{1}+1}{p+n_{1}-1} e^{\varepsilon \tau} \int_{-\tau}^{0}|\mathbf{x}(s)|^{p+n_{1}-1} \mathrm{~d} s \\
& +2 \underline{\kappa} e^{\varepsilon \tau} \int_{-\tau}^{0}|\mathbf{x}(s)|^{p} \mathrm{~d} s \\
& +\frac{p}{2} 5(p-1) \gamma^{2} \frac{2 n_{2}}{p+2 n_{2}-2} e^{\varepsilon \tau} \\
& \times \int_{-\tau}^{0}|\mathbf{x}(s)|^{p+2 n_{2}-2} \mathrm{~d} s
\end{aligned}
$$

$$
\begin{aligned}
& +5(p-1) \gamma^{2} e^{\varepsilon \tau} \int_{-\tau}^{0}|\mathbf{x}(s)|^{p} \mathrm{~d} s \\
& +\int_{0}^{t} e^{\varepsilon s}\left[2 \xi_{1}^{p / 2}(s)+5(p-1) \xi_{2}^{p}(s)\right] \mathrm{d} s .
\end{aligned}
$$

By virtue of the boundedness of $\xi_{1}(t), \xi_{2}(t)$, there is a constant $\Psi>0$ such that $\xi_{1}(t) \vee \xi_{2}(t) \leq \Psi$, which implies that

$$
E e^{\varepsilon t}|\mathbf{x}(t)|^{p} \leq c+\left[2 \Psi^{p / 2}+5(p-1) \Psi^{p}+\frac{p}{2} Q\right] \int_{0}^{t} e^{\varepsilon s} \mathrm{~d} s
$$

where $c=p \bar{\kappa}\left(\left(n_{1}+1\right) /\left(p+n_{1}-1\right)\right) e^{\varepsilon \tau} \int_{-\tau}^{0}|\mathbf{x}(s)|^{p+n_{1}-1} \mathrm{~d} s+$ $(p / 2) 5(p-1) \gamma^{2}\left(2 n_{2} /\left(p+2 n_{2}-2\right)\right) e^{\varepsilon \tau} \int_{-\tau}^{0}|\mathbf{x}(s)|^{p+2 n_{2}-2} \mathrm{~d} s+$ $2 \underline{\kappa} e^{\varepsilon \tau} \int_{-\tau}^{0}|\mathbf{x}(s)|^{p} \mathrm{~d} s+5(p-1) \gamma^{2} e^{\varepsilon \tau} \int_{-\tau}^{0}|\mathbf{x}(s)|^{p} \mathrm{~d} s+E|\mathbf{x}(0)|^{p}$. This implies

$$
\begin{aligned}
E|\mathbf{x}(t)|^{p} \leq & c e^{-\varepsilon t}+\frac{2 \Psi^{p / 2}+5(p-1) \Psi^{p}+(p / 2) Q}{\varepsilon} \\
& \times\left(1-e^{-\varepsilon t}\right) .
\end{aligned}
$$

From the boundedness of initial data $\zeta \in C$, we claim that, for any $p \geq 2$, there exists a constant $M_{p}>0$ such that $\sup _{-\tau \leq t<+\infty} E|\mathbf{x}(t, \zeta)|^{p} \leq M_{p}$. When $p \in(0,2)$, using the Lyapunov inequality, we claim that

$$
\sup _{-\tau \leq t<\infty} E|\mathbf{x}(t)|^{p} \leq\left(\sup _{-\tau \leq t<\infty} E|\mathbf{x}(t)|^{2}\right)^{p / 2} \leq M_{2}^{p / 2}
$$

From Theorem 11 and the Chebyshev inequality, we get the following proposition about the asymptotic boundedness of the global solution in the sense of the trajectory with large probability.

Proposition 12. If Assumptions 1 and 3 and $\kappa>\bar{\kappa}$ hold, then for any initial data $\zeta \in C$, the global solution $\mathbf{x}(t, \zeta)$ of system (5) is stochastically ultimately bounded; namely, for any $\varepsilon \in$ $(0,1)$, there exists a constant $L=L(\varepsilon)>0$ such that

$$
\limsup _{t \rightarrow \infty} P\{|\mathbf{x}(t, \zeta)| \leq L\} \geq 1-\varepsilon
$$

Proof. For any $\varepsilon \in(0,1)$, letting $L=\left(M_{p} / \varepsilon\right)^{1 / p}$, applying Theorem 11 and the Chebyshev inequality, we have

$$
P\{|\mathbf{x}(t, \zeta)|>L\} \leq \frac{E|\mathbf{x}(t, \zeta)|^{p}}{L^{p}} \leq \varepsilon
$$

So we get the required assertion.

Further we continue to discuss the asymptotic boundedness of the norm of $\mathbf{x}_{t}$ in system (5) in the sense of the $p$ th moment and the trajectory with large probability. 
Theorem 13. If Assumptions 1 and 3 and $\kappa>\bar{\kappa}$ hold, then for any initial data $\zeta \in C$, the norm of $\mathbf{x}_{t}$ in system (5) is bounded in the sense of pth moment; that is, there exists a constant $N_{p}>0$ such that the global solution $\mathbf{x}(t, \zeta)$ of system (5) has the property

$$
\sup _{0 \leq t<+\infty} E\left\|\mathbf{x}_{t}\right\|^{p} \leq N_{p}
$$

Proof. For the sake of simplicity, write $\mathbf{x}(t)=\mathbf{x}(t, \zeta)$. From Theorem 11, set $\sup _{-\tau \leq t<\infty} E|\mathbf{x}(t)|^{p} \leq M_{p}$. For $p \geq 2, \quad t \geq 0$, and $\beta \in[0, \tau]$, using Itô's formula, we compute that

$$
\begin{aligned}
& |\mathbf{x}(t-\beta)|^{p} \\
& =|\mathbf{x}(t-\tau)|^{p} \\
& \quad+\int_{t-\tau}^{t-\beta} \frac{p}{2}|\mathbf{x}(s)|^{p-2} \\
& \quad \times\left[2 \mathbf{x}^{T}(s) \mathbf{f}\left(\mathbf{x}_{s}, s\right)+\left|\mathbf{g}\left(\mathbf{x}_{s}, s\right)\right|^{2}\right. \\
& \quad+\int_{t-\tau}^{t-\beta} p|\mathbf{x}(s)|^{p-2} \mathbf{x}^{T}(s) \mathbf{g}\left(\mathbf{x}_{s}, s\right) \mathrm{d} B(s) \\
& =|\mathbf{x}(t-\tau)|^{p}+\frac{p}{2} I_{1} \\
& \quad+\int_{t-\tau}^{t-\beta} p|\mathbf{x}(s)|^{p-2} \mathbf{x}^{T}(s) \mathbf{g}\left(\mathbf{x}_{s}, s\right) \mathrm{d} B(s),
\end{aligned}
$$

where $I_{1}=\int_{t-\tau}^{t-\beta}|\mathbf{x}(s)|^{p-2}\left[2 \mathbf{x}^{T}(s) \mathbf{f}\left(\mathbf{x}_{s}, s\right)+\left|\mathbf{g}\left(\mathbf{x}_{s}, s\right)\right|^{2}+\right.$ $\left.(p-2)|\mathbf{x}(s)|^{-2} \|\left.\mathbf{x}^{T}(s) \mathbf{g}\left(\mathbf{x}_{s}, s\right)\right|^{2}\right] \mathrm{d} s$. Using Young's inequality, we compute that

$I_{1}$

$$
\begin{aligned}
& \leq \int_{t-\tau}^{t-\beta}|\mathbf{x}(s)|^{p-2} \\
& \times\left[2 \mathbf{x}^{T}(s) \mathbf{f}\left(\mathbf{x}_{s}, s\right)+(p-1)\left|\mathbf{g}\left(\mathbf{x}_{s}, s\right)\right|^{2}\right] \mathrm{d} s \\
& \leq \int_{t-\tau}^{t-\beta}|\mathbf{x}(s)|^{p-2} \\
& \times\left[-2 \kappa|\mathbf{x}(s)|^{n_{1}+1}\right. \\
& \quad+2 \bar{\kappa} \int_{-\tau}^{0}|\mathbf{x}(s+\theta)|^{n_{1}+1} \mathrm{~d} \eta_{1}(\theta)-2|\mathbf{x}(s)|^{2} \\
& \quad+2 \underline{\kappa} \int_{-\tau}^{0}|\mathbf{x}(s+\theta)|^{2} \mathrm{~d} \eta_{2}(\theta) \\
& \quad+2 \xi_{1}(s)+5(p-1) \gamma^{2}
\end{aligned}
$$

$$
\begin{aligned}
& \times\left(|\mathbf{x}(s)|^{2 n_{2}}\right. \\
& +\int_{-\tau}^{0}|\mathbf{x}(s+\theta)|^{2 n_{2}} \mathrm{~d} \eta_{3}(\theta)+|\mathbf{x}(s)|^{2} \\
& \left.+\int_{-\tau}^{0}|\mathbf{x}(s+\theta)|^{2} \mathrm{~d} \eta_{4}(\theta)\right) \\
& \left.+5(p-1) \xi_{2}^{2}(s)\right] \mathrm{d} s \\
& \leq \int_{t-\tau}^{t-\beta}\left[(-2 \kappa+2 \bar{\kappa})|\mathbf{x}(s)|^{p+n_{1}-1}\right. \\
& +10(p-1) \gamma^{2}|\mathbf{x}(s)|^{p+2 n_{2}-2} \\
& +\left(-2+2 \underline{\kappa}+10(p-1) \gamma^{2}\right. \\
& \left.+\frac{2(p-2)}{p}+5(p-1) \frac{p-2}{p}\right) \\
& \times|\mathbf{x}(s)|^{p}+2 \bar{\kappa} \frac{n_{1}+1}{p+n_{1}-1} \bar{J}_{1}+2 \underline{\kappa} \frac{2}{p} \bar{J}_{2} \\
& +5(p-1) \gamma^{2} \frac{2 n_{2}}{p+2 n_{2}-2} \bar{J}_{3}+5(p-1) \gamma^{2} \\
& \left.\times \frac{2}{p} \bar{J}_{4}+\frac{4}{p} \xi_{1}^{p / 2}(s)+5(p-1) \frac{2}{p} \xi_{2}^{p}(s)\right] \mathrm{d} s,
\end{aligned}
$$

where $\bar{J}_{1}=\int_{-\tau}^{0}|\mathbf{x}(s+\theta)|^{p+n_{1}-1} \mathrm{~d} \eta_{1}(\theta)-|\mathbf{x}(s)|^{p+n_{1}-1}, \bar{J}_{2}=$ $\int_{-\tau}^{0}|\mathbf{x}(s+\theta)|^{p} \mathrm{~d} \eta_{2}(\theta)-|\mathbf{x}(s)|^{p}, \bar{J}_{3}=\int_{-\tau}^{0}|\mathbf{x}(s+\theta)|^{p+2 n_{2}-2} \mathrm{~d} \eta_{3}(\theta)-$ $|\mathbf{x}(s)|^{p+2 n_{2}-2}$, and $\bar{J}_{4}=\int_{-\tau}^{0}|\mathbf{x}(s+\theta)|^{p} \mathrm{~d} \eta_{4}(\theta)-|\mathbf{x}(s)|^{p}$. However we can get $\int_{t-\tau}^{t-\beta} \bar{J}_{i} \mathrm{~d} s=\int_{t-\tau}^{t-\beta} \int_{-\tau}^{0}|\mathbf{x}(s+\theta)|^{w_{i}} d \eta_{i}(\theta)-|\mathbf{x}(s)|^{w_{i}} \mathrm{~d} s \leq$ $\int_{t-2 \tau}^{t-\tau}|\mathbf{x}(s)|^{w_{i}} \mathrm{~d} s$, for $w_{1}=p+n_{1}-1, w_{3}=p+2 n_{2}-2$, and $w_{2}=w_{4}=p$, respectively.

Let $Q(|\mathbf{x}(t)|)=(-2 \kappa+2 \bar{\kappa})|\mathbf{x}(t)|^{p+n_{1}-1}+10(p-$ 1) $\gamma^{2}|\mathbf{x}(t)|^{p+2 n_{2}-2}+\left(-2+2 \underline{\kappa}+10(p-1) \gamma^{2}+2(p-2) / p+5(p-\right.$ 1) $((p-2) / p))|\mathbf{x}(t)|^{p}$. Since $\kappa>\bar{\kappa}, n_{1}+1>2 n_{2} \geq 2$, by the same technique as (A.3) in the Appendix, we get that there exists a positive constant $\bar{K}$ such that $Q(|\mathbf{x}(t)|) \leq \bar{K}$. By virtue of the boundedness of $\xi_{1}, \xi_{2}$, assuming $\xi_{1}(t) \vee \xi_{2}(t) \leq \Psi$, we have

$$
\begin{aligned}
I_{1} \leq & \bar{K} \tau+2 \bar{\kappa} \frac{n_{1}+1}{p+n_{1}-1} \int_{t-2 \tau}^{t-\tau}|\mathbf{x}(s)|^{p+n_{1}-1} \mathrm{~d} s \\
& +5(p-1) \gamma^{2} \frac{2 n_{2}}{p+2 n_{2}-2} \int_{t-2 \tau}^{t-\tau}|\mathbf{x}(s)|^{p+2 n_{2}-2} \mathrm{~d} s \\
& +\left(2 \underline{\kappa}+5(p-1) \gamma^{2}\right) \frac{2}{p} \int_{t-2 \tau}^{t-\tau}|\mathbf{x}(s)|^{p} \mathrm{~d} s \\
& +\left(\frac{4}{p} \Psi^{p / 2}+5(p-1) \frac{2}{p} \Psi^{p}\right) \tau \\
:= & I_{2} .
\end{aligned}
$$



have

By virtue of the boundedness of $\sup _{-\tau \leq t<\infty} E|\mathbf{x}(t)|^{p}$, we

$$
\begin{aligned}
E\left(I_{2}\right) & \\
= & \bar{K} \tau+2 \bar{\kappa} \frac{n_{1}+1}{p+n_{1}-1} E \int_{t-2 \tau}^{t-\tau}|\mathbf{x}(s)|^{p+n_{1}-1} \mathrm{~d} s \\
& +\left(2 \underline{\kappa}+5(p-1) \underline{\gamma}^{2}\right) \frac{2}{p} E \int_{t-2 \tau}^{t-\tau}|\mathbf{x}(s)|^{p} \mathrm{~d} s \\
& +5(p-1) \bar{\gamma}^{2} \frac{2 n_{2}}{p+2 n_{2}-2} \\
& \times E \int_{t-2 \tau}^{t-\tau}|\mathbf{x}(s)|^{p+2 n_{2}-2} \mathrm{~d} s \\
& +\left(\frac{4}{p} \Psi^{p / 2}+5(p-1) \frac{2}{p} \Psi^{p}\right) \tau \\
\leq & \bar{K}_{\tau}+2 \bar{\kappa} \frac{n_{1}+1}{p+n_{1}-1} M_{p+n_{1}-1} \tau \\
& +5(p-1) \bar{\gamma}^{2} \frac{2 n_{2}}{p+2 n_{2}-2} M_{p+2 n_{2}-2} \tau \\
& +\left(2 \underline{\kappa}+5(p-1) \underline{\gamma}^{2}\right) \frac{2}{p} M_{p} \tau \\
& +\left(\frac{4}{p} \Psi^{p / 2}+5(p-1) \frac{2}{p} \Psi^{p}\right) \tau \\
& \bar{M}_{1} \cdot
\end{aligned}
$$

Therefore, from (26), we have

$$
\begin{aligned}
E \sup _{0 \leq \beta \leq \tau}|\mathbf{x}(t-\beta)|^{p} & \\
\leq & E|\mathbf{x}(t-\tau)|^{p}+\frac{p}{2} \bar{M}_{1} \\
& +\left.E \sup _{0 \leq \beta \leq \tau}\left|\int_{t-\tau}^{t-\beta} p\right| \mathbf{x}(s)\right|^{p-2} \mathbf{x}^{T}(s) \mathbf{g}\left(\mathbf{x}_{s}, s\right) \mathrm{d} B(s) \mid \\
\leq & M_{p}+\frac{p}{2} \bar{M}_{1} \\
& +\left.E \sup _{0 \leq \beta \leq \tau}\left|\int_{t-\tau}^{t-\beta} p\right| \mathbf{x}(s)\right|^{p-2} \mathbf{x}^{T}(s) \mathbf{g}\left(\mathbf{x}_{s}, s\right) \mathrm{d} B(s) \mid .
\end{aligned}
$$

Using B-D-G inequality, we get that

$$
\begin{aligned}
& \left.E \sup _{0 \leq \beta \leq \tau}\left|\int_{t-\tau}^{t-\beta} p\right| \mathbf{x}(s)\right|^{p-2} \mathbf{x}^{T}(s) \mathbf{g}\left(\mathbf{x}_{s}, s\right) \mathrm{d} B(s) \mid \\
& \leq \sqrt{32} E\left[\int_{t-\tau}^{t} p^{2}|\mathbf{x}(s)|^{2 p-4}|\mathbf{x}(s)|^{2}\left|\mathbf{g}\left(\mathbf{x}_{s}, s\right)\right|^{2} \mathrm{~d} s\right]^{1 / 2} \\
& \leq \sqrt{32} p E\left[\sup _{0 \leq \beta \leq \tau}|\mathbf{x}(t-\beta)|^{p} \int_{t-\tau}^{t}|\mathbf{x}(s)|^{p-2}\left|\mathbf{g}\left(\mathbf{x}_{s}, s\right)\right|^{2} \mathrm{~d} s\right]^{1 / 2} \\
& \leq \frac{1}{2} E \sup _{0 \leq \beta \leq \tau}|\mathbf{x}(t-\beta)|^{p}+16 p^{2} E \int_{t-\tau}^{t}|\mathbf{x}(s)|^{p-2}\left|\mathbf{g}\left(\mathbf{x}_{s}, s\right)\right|^{2} \mathrm{~d} s \\
& \leq \frac{1}{2} E \sup _{0 \leq \beta \leq \tau}|\mathbf{x}(t-\beta)|^{p}+\bar{M}_{2},
\end{aligned}
$$

where the boundedness of $E \int_{t-\tau}^{t}|\mathbf{x}(s)|^{p-2}\left|\mathbf{g}\left(\mathbf{x}_{s}, s\right)\right|^{2} \mathrm{~d} s$ can be obtained from the boundedness of $I_{1}$ and $\sup _{-\tau \leq t<\infty} E|\mathbf{x}(t)|^{p}$ above and $\bar{M}_{2}$ is a constant which is not necessary to know exactly. Substituting it into (30), we yield

$$
E\left\|\mathbf{x}_{t}\right\|^{p} \leq 2 M_{p}+p \bar{M}_{1}+2 \bar{M}_{2} .
$$

So we claim that there exists a $N_{p}>0$ such that $\sup _{0 \leq t<\infty} E\left\|\mathbf{x}_{t}\right\|^{p}<N_{p}$ for any $p \geq 2$. When $p \in(0,2)$, using the Lyapunov inequality, we claim that

$$
\sup _{0 \leq t<\infty} E\left\|\mathbf{x}_{t}\right\|^{p} \leq\left(\sup _{0 \leq t<\infty} E\left\|\mathbf{x}_{t}\right\|^{2}\right)^{p / 2} \leq N_{2}^{p / 2} .
$$

Remark 14. Clearly, the key of the proof is the upperboundedness of function $Q(|\mathbf{x}(t)|)$, which depends on the condition $\kappa>\bar{\kappa}$. And the theorem will play an important role to ensure the almost sure asymptotic stability of the solution.

In the same way as Proposition 12, we get the following proposition.

Proposition 15. If Assumptions 1 and 3 and $\kappa>\bar{\kappa}$ hold, then for any initial data $\zeta \in C$, the norm of $\mathbf{x}_{t}$ in system (5) is stochastically ultimately bounded; namely, for any $\varepsilon^{\prime} \in(0,1)$, there exists a constant $L^{\prime}=L^{\prime}\left(\varepsilon^{\prime}\right)>0$ such that the global solution $\mathbf{x}(t, \zeta)$ of system (5) has the property

$$
\limsup _{t \rightarrow \infty} P\left\{\left\|\mathbf{x}_{t}\right\| \leq L^{\prime}\right\} \geq 1-\varepsilon^{\prime}
$$

\section{Almost Sure Asymptotic Stability of SFDEs}

In this section, we aim to study the almost sure asymptotic stability of system (5). The following theorem establishes new criteria on the almost sure asymptotic stability.

Theorem 16. If Assumptions 1 and 3 and the following condition (35) hold,

$$
\begin{gathered}
\frac{\gamma^{2}}{\kappa-\bar{\kappa}}+\frac{\gamma^{2}}{(1-\underline{\kappa})-L(\kappa-\bar{\kappa})}<\frac{1}{2}, \\
(1-\underline{\kappa})-L(\kappa-\bar{\kappa})>0, \quad \kappa-\bar{\kappa}>0,
\end{gathered}
$$

then for any initial data $\zeta \in C$, there is a unique global solution $\mathbf{x}(t, \zeta)$ of system (5) on $t \geq-\tau$, and $\mathbf{x}(t, \zeta)$ is almost surely asymptotically stable; that is,

$$
\lim _{t \rightarrow \infty} \mathbf{x}(t, \zeta)=0 \quad \text { a.s. }
$$

where $L=\left(n_{1}-2 n_{2}+1\right)\left(2 n_{2}-2\right)^{\left(2 n_{2}-2\right) /\left(n_{1}-2 n_{2}+1\right)}\left(n_{1}-\right.$ $1)^{\left(1-n_{1}\right) /\left(n_{1}-2 n_{2}+1\right)}$.

Proof. The existence and uniqueness of the global solution follow from Lemma 9 directly. For the sake of simplicity, write 
$\mathbf{x}(t)=\mathbf{x}(t, \zeta)$. Applying Itô's formula to $V(\mathbf{x}, t)=|\mathbf{x}(t)|^{2}$, we yield

$$
\begin{aligned}
\mathscr{L} V= & 2 \mathbf{x}^{T}(t) \mathbf{f}\left(\mathbf{x}_{t}, t\right)+\left|\mathbf{g}\left(\mathbf{x}_{t}, t\right)\right|^{2} \\
\leq & 2\left[-\kappa|\mathbf{x}(t)|^{n_{1}+1}+\bar{\kappa} \int_{-\tau}^{0}|\mathbf{x}(t+\theta)|^{n_{1}+1} \mathrm{~d} \eta_{1}(\theta)\right. \\
& \left.-|\mathbf{x}(t)|^{2}+\underline{\kappa} \int_{-\tau}^{0}|\mathbf{x}(t+\theta)|^{2} \mathrm{~d} \eta_{2}(\theta)+\xi_{1}(t)\right] \\
& +\frac{1}{\rho_{1}} 2 \gamma^{2}\left(|\mathbf{x}(t)|^{2 n_{2}}+\int_{-\tau}^{0}|\mathbf{x}(t+\theta)|^{2 n_{2}} \mathrm{~d} \eta_{3}(\theta)\right) \\
& +\frac{1}{\rho_{2}\left(1-\rho_{1}\right)} 2 \gamma^{2} \\
& \times\left(|\mathbf{x}(t)|^{2}+\int_{-\tau}^{0}|\mathbf{x}(t+\theta)|^{2} \mathrm{~d} \eta_{4}(\theta)\right) \\
& +\frac{1}{\left(1-\rho_{1}\right)\left(1-\rho_{2}\right)} \xi_{2}^{2}(t) \\
\leq & {\left[-2(\kappa-\bar{\kappa})|\mathbf{x}(t)|^{n_{1}+1}-\left(2-2 \underline{\kappa}-\frac{1}{\rho_{2}\left(1-\rho_{1}\right)} 4 \gamma^{2}\right)\right.} \\
& \left.\times|\mathbf{x}(t)|^{2}+\frac{1}{\rho_{1}} 4 \gamma^{2}|\mathbf{x}(t)|^{2 n_{2}}\right] \\
& +2 \underline{\kappa} \widetilde{J}_{2}+\frac{1}{\rho_{2}\left(1-\rho_{1}\right)} 2 \gamma^{2} \widetilde{J}_{4}+\frac{1}{\rho_{1}} 2 \gamma^{2} \widetilde{J}_{3} \\
& +2 \bar{\kappa} \widetilde{J}_{1}+2 \xi_{1}(t)+\frac{1}{1-\rho_{1}} \frac{1}{1-\rho_{2}} \xi_{2}^{2}(t),
\end{aligned}
$$

where $\rho_{1}, \rho_{2} \in(0,1), \widetilde{J}_{1}=\int_{-\tau}^{0}|\mathbf{x}(t+\theta)|^{n_{1}+1} \mathrm{~d} \eta_{1}(\theta)-|\mathbf{x}(t)|^{n_{1}+1}$, $\widetilde{J}_{2}=\int_{-\tau}^{0}|\mathbf{x}(t+\theta)|^{2} \mathrm{~d} \eta_{2}(\theta)-|\mathbf{x}(t)|^{2}, \widetilde{J}_{3}=\int_{-\tau}^{0}|\mathbf{x}(t+\theta)|^{2 n_{2}} \mathrm{~d} \eta_{3}(\theta)-$ $|\mathbf{x}(t)|^{2 n_{2}}$, and $\widetilde{J}_{4}=\int_{-\tau}^{0}|\mathbf{x}(t+\theta)|^{2} \mathrm{~d} \eta_{4}(\theta)-|\mathbf{x}(t)|^{2}$, and we have used the elemental inequality: for any $c, b \in R, 0<\theta<1$,

$$
(c+b)^{2} \leq \frac{c^{2}}{1-\theta}+\frac{b^{2}}{\theta} .
$$

Let $H(|\mathbf{x}(t)|)=\left(2(1-\underline{\kappa})-\left(4 \gamma^{2} /\left(1-\rho_{1}\right)\right)\right)+2(\kappa-\bar{\kappa})|\mathbf{x}(t)|^{n_{1}-1}-$ $\left(4 \gamma^{2} / \rho_{1}\right)|\mathbf{x}(t)|^{2 n_{2}-2}$. Since $\gamma^{2} /(\kappa-\bar{\kappa})+\gamma^{2} /((1-\underline{\kappa})-L(\kappa-\bar{\kappa}))<$ $1 / 2$, there exists $w_{0}>1$ such that $\left(2 \gamma^{2} /(\kappa-\bar{\kappa})\right) w_{0}+2 \gamma^{2} /((1-$ $\underline{\kappa})-L(\kappa-\bar{\kappa}))=1$. Setting $\rho(w)=\left(2 \gamma^{2} /(\kappa-\bar{\kappa})\right) w, w \in\left(1, w_{0}\right)$, satisfying $\kappa-\bar{\kappa} \geq 2 \gamma^{2} / \rho(w),(1-\underline{\kappa})-2 \gamma^{2} /(1-\rho(w))>(\kappa-$ $\bar{\kappa}) L$. By using Lemma 6 , we get that there exists a constant $\bar{a}>0$ satisfying inf $t \geq 0 H(|\mathbf{x}(t)|)>\bar{a}$. Then choose $\rho_{2}$ which is sufficiently close to 1 such that

$$
\begin{aligned}
& \left(2(1-\underline{\kappa})-\frac{4 \gamma^{2}}{\left(1-\rho_{1}\right) \rho_{2}}\right)-\frac{4 \gamma^{2}}{\rho_{1}}|\mathbf{x}(t)|^{2 n_{2}-2} \\
& +2(\kappa-\bar{\kappa})|\mathbf{x}(t)|^{n_{1}-1}>\bar{a} .
\end{aligned}
$$

We therefore have

$$
\begin{aligned}
\mathscr{L} V \leq & -\bar{a}|\mathbf{x}(t)|^{2}+2 \bar{\kappa} \widetilde{J}_{1}+\frac{2 \gamma^{2}}{\rho_{1}} \widetilde{J}_{3}+2 \underline{\kappa} \widetilde{J}_{2}+\frac{2 \gamma^{2}}{\rho_{2}\left(1-\rho_{1}\right)} \widetilde{J}_{4} \\
& +2 \xi_{1}(t)+\frac{1}{1-\rho_{1}} \frac{1}{1-\rho_{2}} \xi_{2}^{2}(t) .
\end{aligned}
$$

In view of the fact that $\int_{0}^{t} \widetilde{J}_{i} \mathrm{~d} s=\int_{0}^{t}\left(\int_{-\tau}^{0}|\mathbf{x}(s+\theta)|^{w_{i}^{\prime}} \mathrm{d} \eta_{i}(\theta)-\right.$ $\left.|\mathbf{x}(s)|^{w_{i}^{\prime}}\right) \mathrm{d} s \leq \int_{-\tau}^{0}|\mathbf{x}(s)|^{w_{i}^{\prime}} \mathrm{d} s$, for $w_{1}^{\prime}=n_{1}+1, w_{3}^{\prime}=2 n_{2}$, and $w_{2}^{\prime}=w_{4}^{\prime}=2$, respectively, we get

$$
\begin{aligned}
|\mathbf{x}(t)|^{2} \leq & |\mathbf{x}(0)|^{2}-\bar{a} \int_{0}^{t}|\mathbf{x}(s)|^{2} \mathrm{~d} s \\
& +\frac{2 \gamma^{2}}{\rho_{1}} \int_{-\tau}^{0}|\mathbf{x}(s)|^{2 n_{2}} \mathrm{~d} s+2 \bar{\kappa} \int_{-\tau}^{0}|\mathbf{x}(s)|^{n_{1}+1} \mathrm{~d} s \\
& +\left(2 \underline{\kappa}+\frac{2 \gamma^{2}}{\rho_{2}\left(1-\rho_{1}\right)}\right) \int_{-\tau}^{0}|\mathbf{x}(s)|^{2} \mathrm{~d} s \\
& +\int_{0}^{t}\left[\frac{1}{1-\rho_{1}} \frac{1}{1-\rho_{2}} \xi_{2}^{2}(s)+2 \xi_{1}(s)\right] \mathrm{d} s+M(t),
\end{aligned}
$$

where $M(t)=\int_{0}^{t} 2 \mathbf{x}^{T}(s) \mathbf{g}\left(\mathbf{x}_{s}, s\right) \mathrm{d} B(s)$, which is a local martingale with the initial value $M(0)=0$. From Lemma 5 , we get $\int_{0}^{+\infty}\left[\left(1 /\left(1-\rho_{1}\right)\right)\left(1 /\left(1-\rho_{2}\right)\right) \xi_{2}^{2}(s)+2 \xi_{1}(s)\right] \mathrm{d} s<$ $\infty$. Applying the nonnegative semimartingale convergence theorem (see [23]), we obtain that

$$
\limsup _{0 \leq t<\infty}|\mathbf{x}(t)|^{2}<\infty, \quad \int_{0}^{\infty}|\mathbf{x}(t)|^{2} \mathrm{~d} s<\infty \quad \text { a.s. }
$$

To obtain our main result, we need to claim that almost every sample path of $|\mathbf{x}(t)|^{2}$ is uniformly continuous on $[0, \infty)$. Let $|\mathbf{x}(t)|^{2}=|\mathbf{x}(0)|^{2}+\mathbf{y}(t)+\mathbf{z}(t)$, where $\mathbf{y}(t)=\int_{0}^{t}\left(2 \mathbf{x}^{T}(r) \mathbf{f}\left(\mathbf{x}_{r}, r\right)+\left|\mathbf{g}\left(\mathbf{x}_{r}, r\right)\right|^{2}\right) \mathrm{d} r, \mathbf{z}(t)=\int_{0}^{t} 2 \mathbf{x}^{T}(r) \mathbf{g}\left(\mathbf{x}_{r}\right.$, $r) \mathrm{d} B(r)$. From (42), we get that there is a constant $L_{1}>0$ such that $\sup _{0 \leq t<\infty}|\mathbf{x}(t)| \leq L_{1}$. By the boundedness of $|\mathbf{x}(t)|$ and initial data $\zeta$, we get that there is a constant $L_{2}>0$ such that $\sup _{0 \leq t<\infty}\left\|\mathbf{x}_{t}\right\|=\sup _{0 \leq t<\infty,-\tau \leq \theta \leq 0}|\mathbf{x}(t+\theta)| \leq L_{2}$. By virtue of the boundedness of $\xi_{1}(t), \xi_{2}(t)$, assume $\xi_{1}(t) \vee \xi_{2}(t) \leq \Psi$. From Assumption 3, it is easy to conclude that

$$
\begin{aligned}
\mathbf{x}(t)^{T} \mathbf{f}\left(\mathbf{x}_{t}, t\right) \leq & -\kappa|\mathbf{x}(t)|^{n_{1}+1}+\bar{\kappa}\left\|\mathbf{x}_{t}\right\|^{n_{1}+1} \\
& -|\mathbf{x}(t)|^{2}+\underline{\kappa}\left\|\mathbf{x}_{t}\right\|^{2}+\xi_{1}(t), \\
\left|\mathbf{g}\left(\mathbf{x}_{t}, t\right)\right| \leq & \gamma\left(|\mathbf{x}(t)|^{n_{2}}+\left\|\mathbf{x}_{t}\right\|^{n_{2}}+|\mathbf{x}(t)|+\left\|\mathbf{x}_{t}\right\|\right) \\
& +\xi_{2}(t) .
\end{aligned}
$$


Then we get

$$
\begin{aligned}
& |\mathbf{y}(t, \omega)-\mathbf{y}(s, \omega)| \\
& =\left.\left|\int_{s}^{t} 2 \mathbf{x}^{T}(r) \mathbf{f}\left(\mathbf{x}_{r}, r\right)+\right| \mathbf{g}\left(\mathbf{x}_{r}, r\right)\right|^{2} \mathrm{~d} r \mid \\
& \leq \int_{s}^{t} 2\left|\mathbf{x}^{T}(r) \mathbf{f}\left(\mathbf{x}_{r}, r\right)\right|+\left|\mathbf{g}\left(\mathbf{x}_{r}, r\right)\right|^{2} \mathrm{~d} r \\
& \leq \int_{s}^{t} 2\left(\kappa|\mathbf{x}(r)|^{n_{1}+1}+\bar{\kappa}\left\|\mathbf{x}_{r}\right\|^{n_{1}+1}+|\mathbf{x}(r)|^{2}\right. \\
& \left.\quad+\underline{\kappa}\left\|\mathbf{x}_{r}\right\|^{2}+\xi_{1}(r)\right) \\
& \quad+5 \gamma^{2}\left(|\mathbf{x}(r)|^{2 n_{2}}+\left\|\mathbf{x}_{r}\right\|^{2 n_{2}}+|\mathbf{x}(r)|^{2}+\left\|\mathbf{x}_{r}\right\|^{2}\right) \\
& \quad+5 \xi_{2}^{2}(r) \mathrm{d} r \\
& \leq\left[2\left(\kappa L_{1}^{n_{1}+1}+\bar{\kappa} L_{2}^{n_{1}+1}+L_{1}^{2}+\underline{\kappa} L_{2}^{2}+\Psi\right)\right. \\
& \left.+5 \gamma^{2}\left(L_{1}^{2 n_{2}}+L_{2}^{2 n_{2}}+L_{1}^{2}+L_{2}^{2}\right)+5 \Psi^{2}\right](t-s)
\end{aligned}
$$

for any $0 \leq s<t<\infty$. This means $\mathbf{y}(t, \omega)$ is uniformly continuous on $t \geq 0$.

For any $p>2$, using B-D-G inequality, from Theorems 11 and 13 , we get

$$
\begin{aligned}
& E|\mathbf{z}(t, \omega)-\mathbf{z}(s, \omega)|^{p} \\
& =E\left|\int_{s}^{t} 2 \mathbf{x}^{T}(r) \mathbf{g}\left(\mathbf{x}_{r}, r\right) \mathrm{d} B(r)\right|^{p} \\
& \leq\left[\frac{p(p-1)}{2}\right]^{p / 2}|t-s|^{p / 2-1} \int_{s}^{t} E\left|2 \mathbf{x}^{T}(r) \mathbf{g}\left(\mathbf{x}_{r}, r\right)\right|^{p} \mathrm{~d} r \\
& \leq\left[\frac{p(p-1)}{2}\right]^{p / 2} 2^{p}|t-s|^{p / 2-1} \\
& \times \int_{s}^{t} E\left(|\mathbf{x}(r)|\left|\mathbf{g}\left(\mathbf{x}_{r}, r\right)\right|\right)^{p} \mathrm{~d} r \\
& \leq\left[\frac{p(p-1)}{2}\right]^{p / 2} 2^{p}|t-s|^{p / 2-1} \\
& \times \int_{s}^{t} E\left(\gamma \left(|\mathbf{x}(t)|^{n_{2}+1}+|\mathbf{x}(t)|\left\|\mathbf{x}_{t}\right\|^{n_{2}}+|\mathbf{x}(t)|^{2}\right.\right. \\
& \left.\left.+|\mathbf{x}(t)|\left\|\mathbf{x}_{t}\right\|\right)+|\mathbf{x}(t)| \xi_{2}(t)\right)^{p} \mathrm{~d} r \\
& \leq\left[\frac{p(p-1)}{2}\right]^{p / 2} 2^{p}|t-s|^{p / 2-1} \\
& \times \int_{s}^{t} E\left(\gamma|\mathbf{x}(t)|^{n_{2}+1}+\frac{\gamma}{2}\left\|\mathbf{x}_{t}\right\|^{2 n_{2}}+\left(2 \gamma+\frac{1}{2}\right)|\mathbf{x}(t)|^{2}\right. \\
& \left.+\frac{\gamma}{2}\left\|\mathbf{x}_{t}\right\|^{2}+\frac{1}{2} \xi_{2}^{2}(t)\right)^{p} \mathrm{~d} r
\end{aligned}
$$

From the Kolmogorov-Chentsov theorem (see Lemma 8), we obtain that almost every sample path of $z(t)$ is locally but uniformly Hölder-continuous with exponent $\lambda$ for every $\lambda \in(0,(p-2) / 2 p)$. So we have that almost every sample path of $z(t)$ is uniformly continuous. Therefore, we claim that almost every sample path of $|\mathbf{x}(t)|^{2}$ is uniformly continuous on $[0, \infty)$. Then from Barbalat Lemma (see [20]) and (42), we claim that

$$
\lim _{t \rightarrow \infty} \mathbf{x}(t)=0 \quad \text { a.s. }
$$

Remark 17. Clearly, the key of the proof is the positive lower-boundedness of function $H(|\mathbf{x}(t)|)$, which depends on condition (35). Since the positive lower-boundedness of $H(|\mathbf{x}(t)|)$ can guarantee (41), so we can use the nonnegative semimartingale convergence theorem to get the asymptotic stability.

Remark 18. From the proof above, Assumptions 1 and 3 are enough to guarantee the asymptotic stability of system (5). And the coefficients of system (2) do not satisfy the conditions which are similar to Assumptions 2 of [10] or Assumptions 3 of [11]. So compared with [10,11], the three conditions of guaranteeing the asymptotic stability are weakened to the two conditions by this paper.

\section{Example}

In this section, we will discuss some examples to illustrate our results.

Example 1. Let us return to the SFDE (2). We can compute that

$$
\begin{gathered}
\mathbf{x}^{T} \mathbf{f}\left(\mathbf{x}_{t}, t\right) \leq-4|\mathbf{x}(t)|^{4}+\int_{-\tau}^{0}|\mathbf{x}(t+\theta)|^{4} \mathrm{~d} \eta_{1}(\theta)-2|\mathbf{x}(t)|^{2} \\
\left|\mathbf{g}\left(\mathbf{x}_{t}, t\right)\right| \leq|\mathbf{x}(t)|^{5 / 3}+\int_{-\tau}^{0}|\mathbf{x}(t+\theta)| \mathrm{d} \eta_{2}(\theta)
\end{gathered}
$$


So we obtain that $L=\left(n_{1}-2 n_{2}+1\right)\left(2 n_{2}-2\right)^{\left(2 n_{2}-2\right) /\left(n_{1}-2 n_{2}+1\right)}\left(n_{1}-\right.$ $1)^{\left(1-n_{1}\right) /\left(n_{1}-2 n_{2}+1\right)}=4 / 27$, and condition (35) holds. Through Theorems 13 and 16 , the global solution $\mathbf{x}(t, \zeta)$ of system (2) has the following properties:

$$
\begin{gathered}
\sup _{0 \leq t<+\infty} E\left\|\mathbf{x}_{t}\right\|^{p} \leq N_{p}, \quad \forall p \geq 0, \\
\lim _{t \rightarrow \infty} \mathbf{x}(t, \zeta)=0,
\end{gathered}
$$

where $N_{p}$ is some positive constant.

Example 2. Let us consider the scalar SFDE as follows:

$$
\begin{aligned}
\mathrm{d} \mathbf{x}(t)=( & -4 \mathbf{x}^{5}(t)-6 \mathbf{x}(t)+2 D_{1}^{3}\left(\mathbf{x}_{t}\right) \\
& \left.+2 D_{2}\left(\mathbf{x}_{t}\right)+\xi(t)\right) \mathrm{d} t \\
+ & \frac{1}{2}\left(\mathbf{x}^{2}(t)+\mathbf{x}(t)+D_{3}^{2}\left(\mathbf{x}_{t}\right)\right. \\
& \left.+D_{4}\left(\mathbf{x}_{t}\right)+\xi(t)\right) \mathrm{d} B(t)
\end{aligned}
$$

with initial data $\{\mathbf{x}(\theta):-\tau \leq \theta \leq 0\}=\zeta \in C$, where $B(t)$ is a scalar Brownian motion. And $D_{i}$ are bounded linear operators from $C([-\tau, 0] ; R)$ to $R$ satisfying $\left|D_{i}\left(\mathbf{x}_{t}\right)\right| \leq$ $\int_{-\tau}^{0}|\mathbf{x}(t+\theta)| \mathrm{d} \eta_{i}(\theta)$, where $\eta_{i}(\cdot)$ are probability measures on $[-\tau, 0], i=1,2,3,4$.

We compute that

$$
\begin{aligned}
\mathbf{x}^{T} \mathbf{f}\left(\mathbf{x}_{t}, t\right)= & -4|\mathbf{x}(t)|^{6}+2 \mathbf{x}(t)^{T} D_{1}^{3}\left(\mathbf{x}_{t}\right)-6|\mathbf{x}(t)|^{2} \\
& +2 \mathbf{x}(t)^{T} D_{2}\left(\mathbf{x}_{t}\right)+\mathbf{x}(t)^{T} \xi(t) \\
\leq & -4|\mathbf{x}(t)|^{6}+\int_{-\tau}^{0}|\mathbf{x}(t+\theta)|^{6} \mathrm{~d} \eta_{1}(\theta) \\
& -\frac{7}{2}|\mathbf{x}(t)|^{2}+\int_{-\tau}^{0}|\mathbf{x}(t+\theta)|^{2} \mathrm{~d} \eta_{2}(\theta)+\frac{1}{2} \xi^{2}(t) \\
\left|\mathbf{g}\left(\mathbf{x}_{t}, t\right)\right| \leq & \frac{1}{2}\left(|\mathbf{x}(t)|^{2}+|\mathbf{x}(t)|+\left|D_{3}\left(\mathbf{x}_{t}\right)\right|^{2}\right. \\
& \left.\quad+\left|D_{4}\left(\mathbf{x}_{t}\right)\right|+\xi(t)\right) \\
\leq & \frac{1}{2}\left(|\mathbf{x}(t)|^{2}+\int_{-\tau}^{0}|\mathbf{x}(t+\theta)|^{2} \mathrm{~d} \eta_{3}(\theta)\right. \\
& \left.\quad+|\mathbf{x}(t)|+\int_{-\tau}^{0}|\mathbf{x}(t+\theta)| \mathrm{d} \eta_{4}(\theta)+\xi(t)\right)
\end{aligned}
$$

So we obtain that $L=\left(n_{1}-2 n_{2}+1\right)\left(2 n_{2}-2\right)^{\left(2 n_{2}-2\right) /\left(n_{1}-2 n_{2}+1\right)}\left(n_{1}-\right.$ $1)^{\left(1-n_{1}\right) /\left(n_{1}-2 n_{2}+1\right)}=1 / 4$, and condition (35) holds.

If the function $\xi(t)$ is defined by

$$
\xi(t)= \begin{cases}1-6^{n}|t-n|, & t \in\left[n-\frac{1}{6^{n}}, n+\frac{1}{6^{n}}\right], \\ 0, & n=1,2,3, \ldots\end{cases}
$$

or $\xi(t)=e^{-t}$, then it is easy to show that $\xi(t)$ is bounded and that $\int_{0}^{+\infty} \xi(t) \mathrm{d} t=1$. Through Theorem 13, the $p$ th moment of the norm of $\mathbf{x}_{t}$ in system (49) is bounded for any $p \geq 0$; namely, there exists a constant $N_{p}>0$ such that

$$
\sup _{0 \leq t<+\infty} E\left\|\mathbf{x}_{t}\right\|^{p} \leq N_{p} .
$$

Through Theorem 16, we claim that, for any given initial data $\zeta \in C$, the solution of system (49) is almost surely asymptotically stable; that is,

$$
\lim _{t \rightarrow \infty} \mathbf{x}(t, \zeta)=0
$$

\section{Appendix}

Proof of Lemma 9. For any given initial data $\zeta \in C$, by Theorem 3.1 in [15] or Lemma 2.3 in [16], Assumption 1 and conditions $\mathbf{f}(0, t)=0$ and $\mathbf{g}(0, t)=0$ guarantee a unique maximal local solution $\mathbf{x}(t, \zeta)$ to system (5) on $t \in\left[-\tau, \sigma_{\infty}\right)$, where $\sigma_{\infty}$ is the explosion time. Let $k_{0}>0$ be sufficiently large satisfying $\|\zeta\|<k_{0}$. For each integer $k \geq k_{0}$, define the stopping time

$$
\tau_{k}=\inf \left\{t \in\left[0, \sigma_{\infty}\right):|x(t)| \geq k\right\} .
$$

Obviously, $\tau_{k}$ is increasing as $k \rightarrow \infty$. Let $\tau_{\infty}=\lim _{t \rightarrow \infty} \tau_{k}$, so $\tau_{\infty} \leq \sigma_{\infty}$ a.s.; if we can obtain that $\tau_{\infty}=\infty$ a.s., then $\sigma_{\infty}=\infty$ a.s. For the sake of simplicity, write $\mathbf{x}(t)=\mathbf{x}(t, \zeta)$. Using Itô's formula to $V(\mathbf{x}, t)=|\mathbf{x}(t)|^{2}$, we yield

$$
\begin{aligned}
& \mathscr{L} V(\mathbf{x}, t) \\
& =2 \mathbf{x}^{T}(t) \mathbf{f}\left(\mathbf{x}_{t}, t\right)+\left|\mathbf{g}\left(\mathbf{x}_{t}, t\right)\right|^{2} \\
& \leq 2\left(-\kappa|\mathbf{x}(t)|^{n_{1}+1}+\bar{\kappa} \int_{-\tau}^{0}|\mathbf{x}(t+\theta)|^{n_{1}+1} \mathrm{~d} \eta_{1}(\theta)\right. \\
& \left.-|\mathbf{x}(t)|^{2}+\underline{\kappa} \int_{-\tau}^{0}|\mathbf{x}(t+\theta)|^{2} \mathrm{~d} \eta_{2}(\theta)+\xi_{1}(t)\right) \\
& +5\left(\gamma^{2}|\mathbf{x}(t)|^{2 n_{2}}\right. \\
& +\gamma^{2} \int_{-\tau}^{0}|\mathbf{x}(t+\theta)|^{2 n_{2}} \mathrm{~d} \eta_{3}(\theta)+\gamma^{2}|\mathbf{x}(t)|^{2} \\
& \left.+\gamma^{2} \int_{-\tau}^{0}|\mathbf{x}(t+\theta)|^{2} \mathrm{~d} \eta_{4}(\theta)+\xi_{2}^{2}(t)\right) \\
& \leq-2(\kappa-\bar{\kappa})|\mathbf{x}(t)|^{n_{1}+1}+10 \gamma^{2}|\mathbf{x}(t)|^{2 n_{2}} \\
& +\left(10 \gamma^{2}-(2-2 \underline{\kappa})\right)|\mathbf{x}(t)|^{2}+2 \bar{\kappa} \widetilde{J}_{1} \\
& +2 \underline{\kappa} \widetilde{J}_{2}+5 \gamma^{2} \widetilde{J}_{3}+5 \gamma^{2} \widetilde{J}_{4}+2 \xi_{1}(t)+5 \xi_{2}^{2}(t),
\end{aligned}
$$

where $\widetilde{J}_{1}=\int_{-\tau}^{0}|\mathbf{x}(t+\theta)|^{n_{1}+1} \mathrm{~d} \eta_{1}(\theta)-|\mathbf{x}(t)|^{n_{1}+1}, \widetilde{J}_{2}=\int_{-\tau}^{0} \mid \mathbf{x}(t+$ $\theta)\left.\right|^{2} \mathrm{~d} \eta_{2}(\theta)-|\mathbf{x}(t)|^{2}, \widetilde{J}_{3}=\int_{-\tau}^{0}|\mathbf{x}(t+\theta)|^{2 n_{2}} \mathrm{~d} \eta_{3}(\theta)-|\mathbf{x}(t)|^{2 n_{2}}$, and $\widetilde{J}_{4}=\int_{-\tau}^{0}|\mathbf{x}(t+\theta)|^{2} \mathrm{~d} \eta_{4}(\theta)-|\mathbf{x}(t)|^{2}$. 
Noting that $\kappa>\bar{\kappa}, n_{1}+1>2 n_{2} \geq 2$, and $|\mathbf{x}(t)| \geq 0$ for any $t \geq 0$, by Lemma $7, R(|\mathbf{x}(t)|)=-2(\kappa-\bar{\kappa})|\mathbf{x}(t)|^{n_{1}+1}+$ $10 \gamma^{2}|\mathbf{x}(t)|^{2 n_{2}}+\left(10 \gamma^{2}-(2-2 \underline{\kappa})\right)|\mathbf{x}(t)|^{2}$, as a function of $|\mathbf{x}(t)|$, has a positive upper-boundedness; that is, there is a positive constant $\widetilde{R}$ such that

$$
\begin{aligned}
R(|\mathbf{x}(t)|)= & -2(\kappa-\bar{\kappa})|\mathbf{x}(t)|^{n_{1}+1}+10 \gamma^{2}|\mathbf{x}(t)|^{2 n_{2}} \\
& +\left(10 \gamma^{2}-(2-2 \underline{\kappa})\right)|\mathbf{x}(t)|^{2} \leq \widetilde{R} .
\end{aligned}
$$

(This technique has been used by many researchers, e.g., [10, 14].)

From Lemma 5, we have $\int_{0}^{+\infty}\left(5 \xi_{2}^{2}(s)+2 \xi_{1}(s)\right) \mathrm{d} s<\infty$. And in view of the fact that

$$
\begin{aligned}
& \int_{0}^{t \wedge \tau_{k}} \widetilde{J}_{i} \mathrm{~d} s \\
& \quad=\int_{0}^{t \wedge \tau_{k}}\left(\int_{-\tau}^{0}|\mathbf{x}(s+\theta)|^{w_{i}^{\prime}} \mathrm{d} \eta_{i}(\theta)-|\mathbf{x}(s)|^{w_{i}^{\prime}}\right) \mathrm{d} s \\
& \quad \leq \int_{-\tau}^{0}|\mathbf{x}(s)|^{w_{i}^{\prime}} \mathrm{d} s
\end{aligned}
$$

for $w_{1}^{\prime}=n_{1}+1, w_{3}^{\prime}=2 n_{2}$, and $w_{2}^{\prime}=w_{4}^{\prime}=2$, we yield that, for $t \geq 0$,

$$
\begin{aligned}
& E\left|\mathbf{x}\left(t \wedge \tau_{k}\right)\right|^{2} \\
& =E|\mathbf{x}(0)|^{2}+E \int_{0}^{t \wedge \tau_{k}}\left[2 \mathbf{x}^{T}(s) \mathbf{f}\left(\mathbf{x}_{s}, s\right)+\left|\mathbf{g}\left(\mathbf{x}_{s}, s\right)\right|^{2}\right] \mathrm{d} s \\
& \leq E|\mathbf{x}(0)|^{2} \\
& \quad+E \int_{0}^{t \wedge \tau_{k}}\left[\widetilde{R}+2 \widetilde{\kappa}_{1}+2 \underline{\kappa} \widetilde{J}_{2}+5 \gamma^{2} \widetilde{J}_{3}\right. \\
& \leq E|\mathbf{x}(0)|^{2}+\widetilde{R} E\left(t \wedge \tau_{k}\right) \\
& \quad+2 \bar{\kappa} \int_{-\tau}^{0}|\mathbf{x}(s)|^{n_{1}+1} \mathrm{~d} s+2 \underline{\kappa} \int_{-\tau}^{0}|\mathbf{x}(s)|^{2} \mathrm{~d} s \\
& \quad+5 \gamma^{2} \int_{-\tau}^{0}|\mathbf{x}(s)|^{2 n_{2}} \mathrm{~d} s+5 \gamma^{2} \int_{-\tau}^{0}|\mathbf{x}(s)|^{2} \mathrm{~d} s \\
& \quad+\int_{0}^{\infty}\left[2 \xi_{1}(s)+5 \xi_{2}^{2}(s)\right] \mathrm{d} s \\
& \leq \bar{R}+\widetilde{R} t,
\end{aligned}
$$

where $\bar{R}=E|\mathbf{x}(0)|^{2}+2 \bar{\kappa} \int_{-\tau}^{0}|\mathbf{x}(s)|^{n_{1}+1} \mathrm{~d} s+2 \underline{\kappa} \int_{-\tau}^{0}|\mathbf{x}(s)|^{2} \mathrm{~d} s+$ $5 \gamma^{2} \int_{-\tau}^{0}|\mathbf{x}(s)|^{2 n_{2}} \mathrm{~d} s+5 \gamma^{2} \int_{-\tau}^{0}|\mathbf{x}(s)|^{2} \mathrm{~d} s+\int_{0}^{\infty}\left[2 \xi_{1}(s)+5 \xi_{2}^{2}(s)\right] \mathrm{d} s$. Noting that

$$
E\left|\mathbf{x}\left(t \wedge \tau_{k}\right)\right|^{2} \geq E\left(\left|\mathbf{x}\left(t \wedge \tau_{k}\right)\right|^{2} I_{\left\{\tau_{k} \leq t\right\}}\right) \geq k^{2} P\left\{\tau_{k} \leq t\right\},
$$

we get that

$$
P\left\{\tau_{\infty} \leq t\right\}=\lim _{k \rightarrow \infty} P\left\{\tau_{k} \leq t\right\} \leq \lim _{k \rightarrow \infty} \frac{\bar{R}+\widetilde{R} t}{k^{2}}=0 .
$$

Since $t$ is arbitrary, we must have that $\tau_{\infty}=\infty$ a.s. and this completes the proof.

\section{Conflict of Interests}

The authors declare that there is no conflict of interests regarding the publication of this paper.

\section{Acknowledgments}

This research was supported by the National Science Foundation of China (no. 11171010), Beijing National Science Foundation (no. 1112001), and Tangshan Science and Technology Bureau Program of Hebei Province (no. 13130214z).

\section{References}

[1] J. Cao and F. Ren, "Exponential stability of discrete-time genetic regulatory networks with delays," IEEE Transactions on Neural Networks, vol. 19, no. 3, pp. 520-523, 2008.

[2] V. Kolmanovskii and A. Myshkis, Applied Theory of Functional Differential Equations, Kluwer Academic, 1992.

[3] J. Liang and J. Lam, "Robust state estimation for stochastic genetic regulatory networks," International Journal of Systems Science, vol. 41, no. 1, pp. 47-63, 2010.

[4] X. Mao and C. Yuan, Stochastic Differential Equations with Markovian Switching, Imperial College Press, London, UK, 2006.

[5] X. Mao, Stochastic Differential Equations and Applications, Horwood Publishing, Chichester, UK, 2nd edition, 2007.

[6] J. A. D. Appleby and D. W. Reynolds, "Decay rates of solutions of linear stochastic Volterra equations," Electronic Journal of Probability, vol. 13, pp. no. 30, 922-943, 2008.

[7] M. Basin and A. Rodkina, "On delay-dependent stability for a class of nonlinear stochastic systems with multiple state delays," Nonlinear Analysis: Theory, Methods \& Applications, vol. 68, no. 8, pp. 2147-2157, 2008.

[8] W. Chen, Z. Guan, and X. Lu, "Delay-dependent exponential stability of uncertain stochastic systems with multiple delays: an LMI approach," Systems \& Control Letters, vol. 54, no. 6, pp. 547-555, 2005.

[9] Y. Chen and A. Xue, "Improved stability criterion for uncertain stochastic delay systems with nonlinear uncertainties," Electronics Letters, vol. 44, no. 7, pp. 458-459, 2008.

[10] L. Liu, Y. Shen, and F. Jiang, "The almost sure asymptotic stability and $p$ th moment asymptotic stability of nonlinear stochastic differential systems with polynomial growth," IEEE Transactions on Automatic Control, vol. 56, no. 8, pp. 1985-1990, 2011.

[11] L. Liu and Y. Shen, "The almost sure asymptotic stability and pth moment asymptotic stability of nonlinear stochastic delay differential systems with polynomial growth," Asian Journal of Control, vol. 15, no. 3, pp. 1-9, 2012.

[12] Q. Luo, X. Mao, and Y. Shen, "Generalised theory on asymptotic stability and boundedness of stochastic functional differential equations," Automatica, vol. 47, no. 9, pp. 2075-2081, 2011. 
[13] Y. Shen, Q. Luo, and X. Mao, "The improved LaSalle-type theorems for stochastic functional differential equations," Journal of Mathematical Analysis and Applications, vol. 318, no. 1, pp. 134154, 2006.

[14] F. Wu and S. Hu, "Stochastic suppression and stabilization of delay differential systems," International Journal of Robust and Nonlinear Control, vol. 21, no. 5, pp. 488-500, 2011.

[15] D. Xu, X. Wang, and Z. Yang, "Further results on existenceuniqueness for stochastic functional differential equations," Science China: Mathematics, vol. 56, no. 6, pp. 1169-1180, 2013.

[16] D. Xu, B. Li, S. Long, and L. Teng, "Moment estimate and existence for solutions of stochastic functional differential equations," Nonlinear Analysis. Theory, Methods \& Applications, vol. 108, pp. 128-143, 2014.

[17] R. Yang, P. Shi, and H. Gao, "New delay-dependent stability criterion for stochastic systems with time delays," IET Control Theory \& Applications, vol. 2, no. 11, pp. 966-973, 2008.

[18] X. Zhao and F. Deng, "Moment stability of nonlinear stochastic systems with time-delays based on calH-representation technique," IEEE Transactions on Automatic Control, vol. 59, no. 3, pp. 814-819, 2014.

[19] X. Mao, "Numerical solutions of stochastic functional differential equations," LMS Journal of Computation and Mathematics, vol. 6, pp. 141-161, 2003.

[20] V. Popov, Hyperstability of Control System, Springer, Berlin, Germany, 1997.

[21] I. Karatzas and S. E. Shreve, Brownian Motion and Stochastic Calculus, Springer, New York, NY, USA, 1991.

[22] S. Fang and T. Zhang, "A study of a class of stochastic differential equations with non-Lipschitzian coefficients," Probability Theory and Related Fields, vol. 132, no. 3, pp. 356-390, 2005.

[23] R. S. Liptser and A. N. Shiryayev, Theory of Martingales, Kluwer Academic Publishers, Dordrecht, The Netherlands, 1989. 


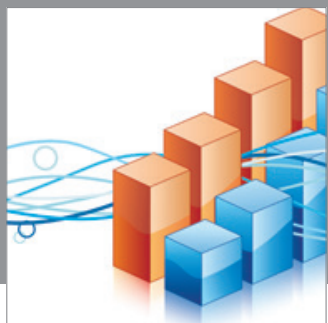

Advances in

Operations Research

mansans

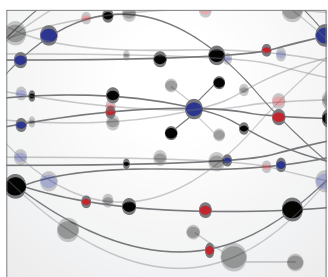

The Scientific World Journal
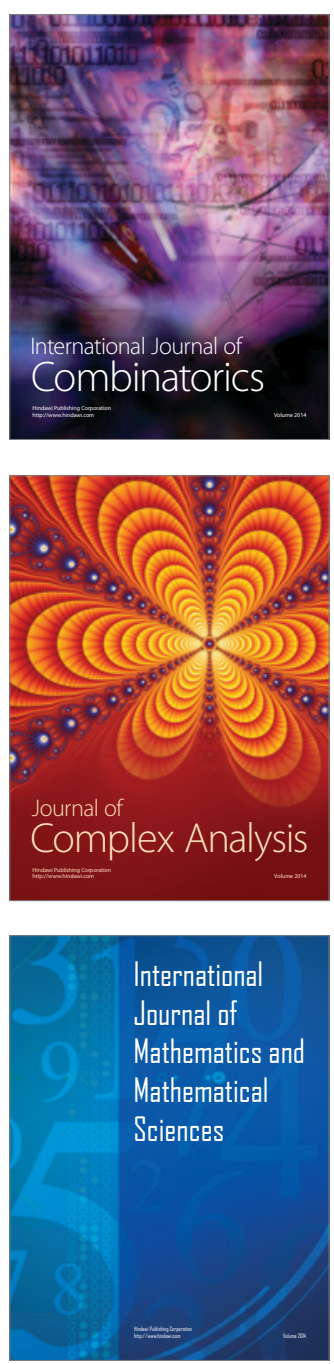
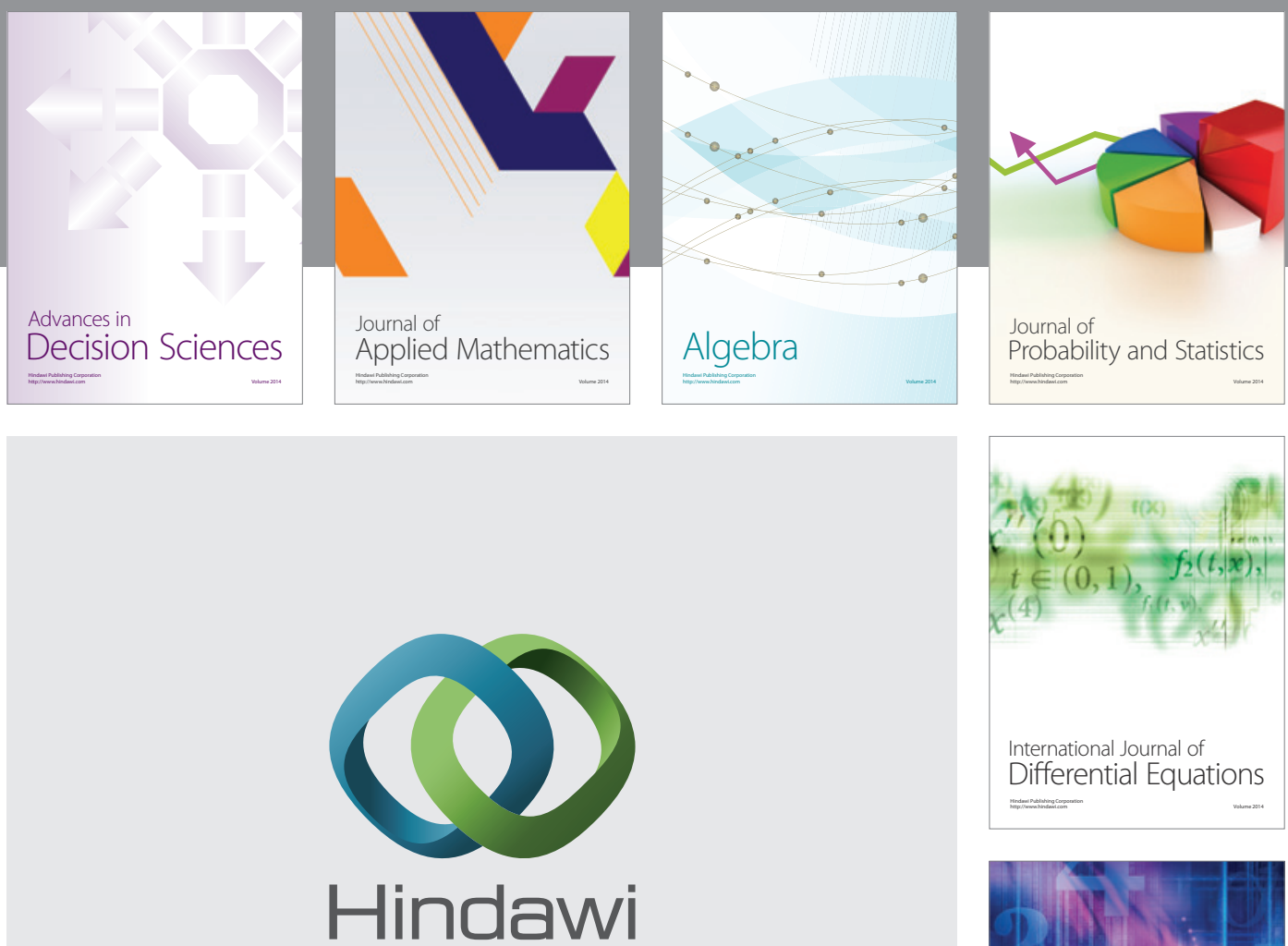

Submit your manuscripts at http://www.hindawi.com
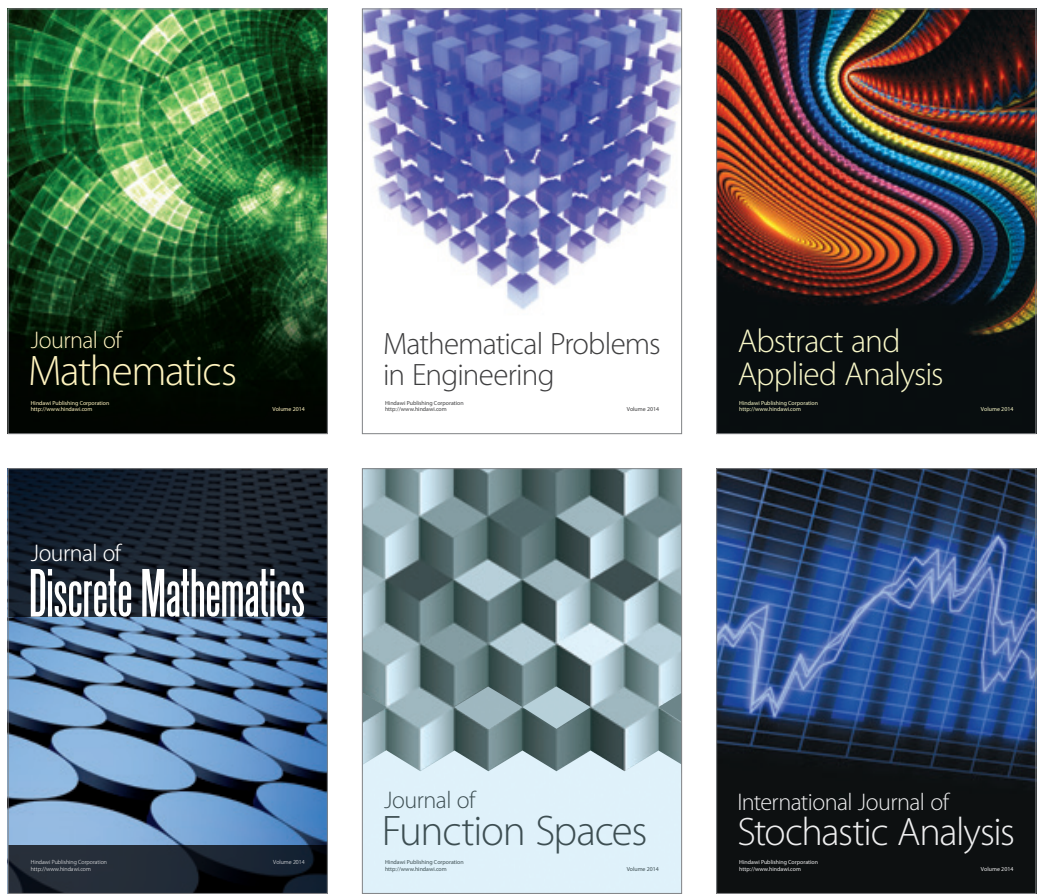

Journal of

Function Spaces

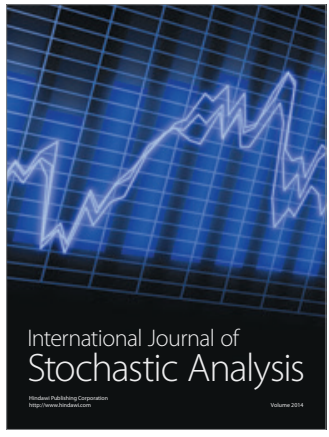

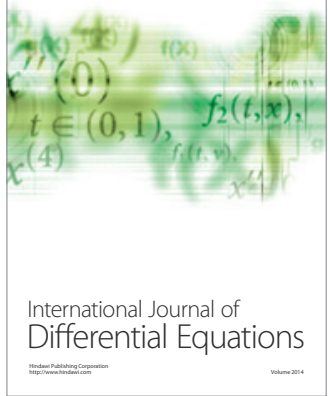
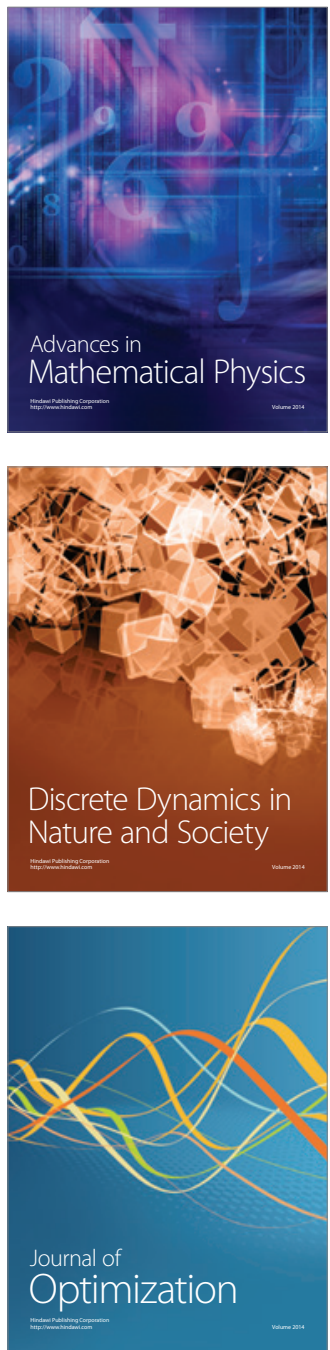